\title{
Fungal septins: one ring to rule it all?
}

Alberto González-Novo ${ }^{1}$, Carlos R. Vázquez de Aldana ${ }^{1}$, Javier Jiménez ${ }^{2}$.

1: Dpto. Microbiología y Genética, Instituto de Microbiología Bioquímica. Universidad de Salamanca /CSIC, Avda. Doctores de la Reina s/n. 37007 Salamanca, Spain. ${ }^{2}$ : Cell Signaling Unit, Departament de Cìnces Experimentals i de la Salut; Universitat Pompeu Fabra; Dr. Aiguader 88 08003. Barcelona, Spain

* Corresponding author: Javier Jiménez 


\begin{abstract}
Septins are a conserved family of GTP-binding proteins found in living organisms ranging from yeasts to mammals. They are able to polymerize and form hetero-oligomers that assemble into higher-order structures, whose detailed molecular architecture has recently been described in different organisms. In Saccharomyces cerevisiae, septins exert numerous functions throughout the cell cycle, serving as scaffolds for many different proteins or as diffusion barriers at the bud neck. In other fungi, septins are required for the proper completion of diverse functions such as polarized growth or pathogenesis. Recent results from several fungi have revealed important differences in septin organization and regulation as compared with $S$. cerevisiae, especially during Candida albicans hyphal growth and in Ashbya gossypii. Here we focus on these recent findings, their relevance in the biology of these eukaryotes and in consequence the "renaissance" of the study of septin structures in cells showing a different kind of morphological behaviour.
\end{abstract}




\section{INTRODUCTION}

Initially identified by Hartwell in 1971 [1] in a search for S. cerevisiae mutants affected in bud emergence and cytokinesis, the septins are a family of GTP-binding proteins that can be found mainly in yeast and animal cells. All septins share structural similarities, and their functions in the different organisms studied to date are also conserved, serving as scaffolds for other proteins and acting as diffusion barrier for proteins associated to cellular membranes. Composed of several subunits, the architecture of the septin complex is not trivial: the different septin monomers assemble into oligomers, which in turn form higher-order structures. These structures are composed of different numbers of subunits, depending on the organism. In $S$. cerevisiae, five septins are present during vegetative growth; i.e. Cdc3, Cdc10, $\mathrm{Cdc} 11, \mathrm{Cdc} 12$ and Shs1 [1-3]. In addition, when the sporulation program is triggered, two of them -Cdc12 and Shs1- are replaced by another two sporulation-specific septins: Spr3 and Spr28 [4, 5].

Although initially discovered through their involvement in cytokinesis, members of the septin family have been implicated in a variety of processes in fungi and in animal cells. Yeast septins have been shown to function as a scaffold for many other proteins, organizing the division site and coordinating nuclear and cellular division. Septins have also been shown to act as a barrier, preventing the diffusion of membrane proteins between mother and daughter cells (for reviews, see [6-8]). In mammalian cells, recent findings have shed light on the dynamics and regulation of septin assembly and have shown that septins are important in processes ranging from actin and microtubule organization to exocytosis and to the maintenance of cell shape. Furthermore, defects in septins have been implicated in several human diseases (for recent reviews on mammalian septins, see [9-14]). Moreover, these proteins are subject to different post-transcriptional modifications, indicating that a finely tuned regulation of septins is crucial for cells. In this review we shall focus on recent findings, both structural and functional, in the field of septins in different fungal organisms that undergo different patterns of growth and differentiation in comparison with $S$. cerevisiae, thus revealing new aspects of septin function. The reader is referred to other excellent recent reviews that cover different aspects of septin biology $[6-8,15-21]$. 
The structure of septins and their domain organization is highly conserved in all organisms studied. Most of them contain a P-loop GTP-binding domain close to their $\mathrm{N}$ terminus that contains several of the conserved motifs common to all P-loop GTPases [22]. Cterminal to the GTPase domain, a septin sequence conserved among the various septins has been identified [23]. Frequently, a poly-basic domain related to phospholipid binding is present at the $\mathrm{N}$ terminus, before the GTP-binding domain [24, 25]. Most septins also have a C-terminal extension predicted to form coiled-coils, which are required for interactions among different septins $[19,24,26]$. A recent evolutionary analysis of septins across kingdoms has indicated that they can be classified in five different groups and that members of Group 1A (which contains S. cerevisiae Cdc10) do not have the C-terminal coiled-coil [27].

More than 30 years ago, electron microscopy studies carried out in budding yeast revealed the presence of so-called "10-nm filaments" surrounding the bud neck [28]. Later, immunofluorescence and GFP-tagging of septin subunits showed that septins have a cell cycledependent localization: in unbudded cells, they form a cap at the future budding site, which later develops into a ring through which bud emergence occurs. As the bud grows, the septin ring changes to a collar that expands the bud neck and, at the time of cytokinesis, the collar splits into two septin rings: one located in the mother cell and the other in the daughter cell. Finally, the septin ring starts to disassemble and a new septin cap marks the budding site for the next cell cycle (Figure 1A). All these transitions are finely regulated and coordinated with cell-cycle progression through the action of multiple signalling pathways. Thus, cap formation requires the Cdc42 GTPase, which carries out a specific role in septin ring formation that is independent of its role in actin polarization [29-31]. Proteins such as Bni5 and Elm1, as well as the phosphorylation of septins by the Cla4 PAK kinase and Gin4, are necessary for collar formation [2, 32-38] (Figure 1A). This phosphorylation is maintained until the onset of cytokinesis, when septins are dephosphorylated by the phosphatase PP2A [39]. Finally, septin ring disassembly after cytokinesis requires the SUMOylation of Cdc3, Cdc11 and Shs1, as well as Cdc3 phosphorylation by Cdc28 [40, 41]. In addition to these classic well-defined localizations, very recently it has been shown that in response to certain nutritional limitations septins disassemble 
from the bud neck and move to and associate with the spindle, both in mitotic and meiotic cells [42]. The exact nature of this localization is not yet known, but it could be a mechanism for septin storage until optimal nutrient conditions are recovered, allowing cells to rapidly reuse septins in order to start growing.

Although the cell cycle-dependent localization of septins has been known for many years, an intriguing question remained to be elucidated: are the septin subunits that disassemble from the neck after cytokinesis recycled to form the new septin structures, or are the new structures composed of septins synthesized de novo? Recently, the answer to this issue was provided by McMurray and Thorner [43], who used a set of very elegant fluorescent pulse-and-chase experiments to demonstrate that septin subunits are highly stable, and that after septin ring disassembly, they are reused in subsequent cell cycles mixed with newly synthesized subunits (see also [44]). This stability is also maintained during meiosis, indicating that it is not dependent on the morphogenetic program.

The ability of septins to polymerize in vitro into filaments that can be observed by electron microscopy is well known [45-49]. However, the exact structure of the filaments and how the different monomers are arranged in them has been a mystery that we are only starting to understand thanks to recent findings on mammalian, worm, and yeast septins. A major breakthrough in our understanding of septin organization was the determination of the crystal structure of human septin complexes [50], which was soon followed by the resolution of the arrangement of $C$. elegans septin complexes by EM [51]. Human septin complexes have a basic building block composed of the Sept2, Sept6 and Sept7 septins. The heterotrimers associate head-to-head to form a hexameric unit that is non-polarized along the filament axis. Interestingly, the coiled-coil domains are arranged away from the filament core, and this could be important for the interaction between different filaments (for reviews on septin structure and assembly, see $[52,53])$. A similar non-polar organization has been found for the C. elegans and S. cerevisiae septin complexes [49-51]. In budding yeast, the basic polymerization unit is a heterotetramer containing a copy of each of the Cdc3, Cdc10, Cdc11 and Cdc12 septins. Yeast septin heterooligomers are composed of eight subunits, and the order of assembly in the 
heterooctamer is Cdc11-Cdc12-Cdc3-Cdc10-Cdc10-Cdc3-Cdc12-Cdc11, also resulting in nonpolar structures. The octamers can then bind through Cdc11 to assemble into non-polar filaments. These studies indicate that the general structure of septin complexes is common to all eukaryotic organisms.

Even though the above results constitute a major advance in our understanding of septin architecture, there are still several questions to be addressed in the future. First, the experiments were performed with recombinant proteins expressed in Escherichia coli, which raises the question of whether they truly reflect the organization of septin complexes in vivo. Interestingly, recent findings using in vivo approaches seem to confirm (at least partially) such a notion [54], suggesting that in fact this may well be the true architecture of septin complexes in budding yeast. In addition, the exact role of the fifth mitotic septin, Shs1, in the structure and function of septin complexes must be clearly elucidated. The analysis of $S$. cerevisiae septins was performed using only four of them, so currently it is unknown how Shs1 is assembled in the septin filaments. Although in wild-type cells Shs1 is not necessary for the assembly of the other septins into the ring, it is important for cytokinesis, and its C-terminal extension is required for septin organization in the absence of other septin monomers [55]. This suggests that if some other septin were missing, i.e. Cdc11, Shs1 would replace it $[49,55]$. Undoubtedly, other open fields of research in the future will be to unravel the role of the physiological inputs and regulatory signals that influence septin organization at different moments of the cell cycle or under specific growth conditions, or to understand how asymmetry in the mother-daughter axis is generated by the non-polar septin filaments.

An additional level of complexity comes from the dynamic behaviour of septin rings in vivo. Fluorescence Recovery After Photobleaching (FRAP) experiments have shown that septin subunits can move around freely inside the ring during certain moments of the cell cycle, i.e. bud emergence and cytokinesis, resulting in a "fluid" state, while during the rest of the cell cycle they cannot diffuse, generating a "frozen" state. Importantly, no replacement of septin subunits by those in the cytoplasm has ever been observed (Figure 1A and $[39,56]$ ). The fine coordination of these dynamic changes is achieved -at least in part- through the regulation of the 
phosphorylation state of one of the septin subunits, Shs1 (which, interestingly, is the only septin whose role in septin complexes formation is still enigmatic), which is phosphorylated by Gin4 and Cla4, and which is dephosphorylated by the PP2A phosphatase requiring its regulatory subunit Rts1 [39], the latter modification being responsible for the "fluid" state during cytokinesis (Figure 1A). In addition to these changes in the dynamic properties of the septin subunits, the septin ring itself shows dynamic behaviour. Thus, in elegant experiments with polarized fluorescence microscopy Vrabioiu and Mitchison demonstrated that in the collar, septin filaments are oriented parallel to the mother-bud axis, and that during cytokinesis they rotate $90^{\circ}$ and change to an orientation parallel to the septum between the two cells (Figure 1B) [57]. How can all these results concerning the dynamic properties of septin subunits and structures be correlated with the architecture of septin complexes? This is an interesting question that should be elucidated in future studies.

\section{SEPTIN FUNCTIONS IN FUNGI}

Knowledge of septin biology is more advanced in S. cerevisiae than in any other fungi, but in recent years a number of studies addressing fungal septins have started to shed light on the function of these proteins in many other organisms. Since many of these fungi have different growth patterns from that of $S$. cerevisiae, such as $C$. albicans filamentous growth or asexual conidiospore formation in A. nidulans, these studies have begun to reveal new aspects of septin function and regulation hitherto not seen in S. cerevisiae. We shall start with a brief overview of septin function in S. cerevisiae, later highlighting the differences with other fungi.

\section{Saccharomyces cerevisiae}

Septins were originally identified in a search for mutants affected in bud emergence and cytokinesis, but with time descriptions of functions reported to be dependent on septins increased rapidly. All such functions are due to two different properties of septins: a) they serve as a scaffold for many other proteins at the bud neck, resulting in the development of certain functions at the bud neck region, and b) septins assemble a diffusion barrier, preventing 
uncontrolled diffusion of plasma membrane-associated proteins between mother and daughter cells (for recent reviews on $S$. cerevisiae septin functions, see $[13,19,52,58,59]$ ).

The first category of functions results in the establishment of a spatial landmark in the cell cortex that is maintained throughout the cell cycle. This landmark allows the recruitment of polarity markers and budding proteins to the incipient budding site $[8,17,60-65]$. Later on in the cell cycle, septins determine the plane of nuclear division and are also essential for the proper migration of the nucleus into the daughter cell in mitosis, since they serve as an interaction point between the mitotic spindle and the bud neck through as yet unknown protein/s [66-68]. Moreover, septins are of key importance in the morphogenesis checkpoint, which is responsible for the coordination between correct bud neck morphology and cell-cycle progression through Hsl1, Hsl7 and the Swe1 kinase [32, 58, 69-74].

It is also known that septins are required for the generation of asymmetry in budding yeast. They are essential for the asymmetric localization of proteins at both sides of the septation plane. Examples are Bni4, which is restricted to the mother side of the neck, or the kinase Kcc4, which is conversely localized at the bud side, which in turn results in a differential function of these proteins [75]. How this asymmetry is achieved is not known, but mutations that alter septin structures before bud emergence abolish this asymmetrical localization, suggesting that the septin structure itself could be asymmetric or composed of polar filaments. However, as mentioned above septin filaments are non-polar, and recent findings using microscopy approaches have suggested that the arrangement of septin subunits in the ring is not asymmetric $[43,76]$. Thus, asymmetry generation must be achieved through other mechanisms. Interestingly, it has been shown that Cdc3, Cdc11 and Shs 1 are modified by SUMOylation, and that this modification is asymmetric, affecting only the mother side of the septin ring [40]. Clearly, studies to uncover asymmetry-generating mechanisms dependent on septins will be one of the fields of interest in future years.

The communication between mother and daughter cell must occur through the bud neck. To maintain the asymmetry between the mother and daughter cells, it is very important that the proper direction of signalling pathways be ensured, thus avoiding incorrect signalling. One 
mechanism that cells have developed is the generation of a diffusion barrier at the bud neck, which blocks the free movement of proteins from one cell to the other. This barrier function at the plasma membrane level is carried out by septins. The correct functioning of this structure ensures the asymmetric distribution of proteins restricting their movement between the two cell bodies and generating polarity in the cells $[66,77-79]$. At the onset of cytokinesis, septin ring division generates a double diffusion barrier between the mother and daughter cells, creating a specialized compartment for cytokinesis. This closed space guarantees, first that the septum biogenesis machinery, and later the hydrolytic enzymes required for controlled dissolution of the septum, will be restricted to the bud neck region [80]. Recently, the group of Dr. Barral has unveiled some surprising roles of the diffusion barrier that are dependent on septins and on the septin-associated protein Bud6 $[81,82]$. They have shown that septins regulate the diffusion of plasma membrane-associated proteins and of endoplasmic reticulum (ER) membrane proteins, generating separable compartments. In contrast, luminal ER proteins are able to move freely between mother and daughter [81]. More recently, the same group has reported that the diffusion barrier also affects the nuclear envelope during anaphase, and as a consequence the daughter cell only inherits newly synthesized nuclear pores [82]. The biological implications of this observation are impressive, since old nuclear pore complexes have associated noncentromeric episomes, which contribute to ageing. Thus, this barrier is not only a system for maintaining asymmetry, but it also plays an essential role in restricting the ageing process to the mother cell, resetting the ageing of the bud and rejuvenating the cellular progeny (see also [20]).

\section{Candida albicans}

Following the pioneering studies in S. cerevisiae by Byers and Goetsch [28], 10-nm filament structures were also observed in the bud neck of yeast cells of the polymorphic human fungal pathogen $C$. albicans [83]. More recently, sequencing of the genome of this fungus revealed the presence of seven septin-coding genes. Both nucleotide and amino acid sequences showed a high degree of conservation with their budding yeast counterparts and were therefore designated as in S. cerevisiae. The generation of null mutants in the genes coding for the seven 
septins revealed that two of them $(C D C 3$ and $C D C 12)$ are essential for cell viability, while the sporulation-specific orthologues showed no expression under the conditions assayed [84]. Mutants in $C D C 10$ and $C D C 11$ have morphological defects (see below), while the sep $7 \triangle$ mutant has been described to have no relevant phenotype, although it was later shown that Sep7 is important in hyphal morphogenesis (see below and $[84,85]$ ).

Early studies on C. albicans septins used immunofluorescence to localize Cdc11 in yeast cells, revealing a localization pattern that was indistinguishable from that found in $S$. cerevisiae, as regards both position and cell-cycle coordination [86]. These observations were later confirmed using in vivo observations of GFP tagged-septins [84, 87]. However, the most remarkable morphogenetic trait of C. albicans is its ability to switch from the yeast to hyphal growth in response to certain external stimuli, this phenomenon being of crucial relevance for pathogenesis [88-90]. Hyphal growth is characterized by certain specific morphogenetic traits that are different from yeast growth (for reviews on C. albicans biology, see [91, 92]). First, hyphae grow continuously in a polarized fashion. This is reflected in the pattern of actin polarization, since actin cortical patches cluster continuously at the growing tip and cables are oriented towards the tip. Second, during hyphal growth cell separation between the different compartments is not activated, allowing the formation of septated filaments. Finally, another peculiarity of hyphal growth is related to septin rings, since they do not disassemble immediately after cytokinesis but remain at both sides of the septum. For this reason, more than one ring can be observed in long hyphae.

When yeast cells are induced to develop filaments, three types of septin structures can be found (Figure 2). Upon germ tube emission, the septins assemble a diffuse structure known as the basal band, consisting of parallel bars. Later, the first hyphal septin ring is not assembled at the junction between the cell body and the germ tube, but 10-15 $\mu \mathrm{m}$ inside the germ tube. Septins can also be found as a faint cap at the tip of the growing hypha $[84,86]$. These hyphalspecific localizations of septins serve as determinants of true hyphal growth, allowing it to be distinguished from the sometimes very similar pseudohyphal growth [92]. 
The role of septins in S. cerevisiae morphogenesis is well known, and hence although it would initially be tempting to conclude that they play similar roles in C. albicans, some interesting differences between these two yeasts have been described. Septin-null mutants in CDC10 and CDC11 show morphological defects, such as abnormal bud shape or aberrant chitin deposition. Additionally, although $c d c 10 \Delta$ and $c d c 11 \Delta$ mutants are able to emit germ tubes, the hyphae have an abnormally curved morphology, indicating that septins are important to maintain a unidirectional, stable cell polarity [84]. In accordance with these morphogenetic defects, septin mutants are also defective for pathogenesis in vivo, as judged by their defects in organ colonization or their reduced ability to kill infected mice, and in vitro, defects in epithelial cell adhesion being observed. Moreover, septin mutants also show defects in invasive growth and severe defects in the general cell wall structure [93, 94], reinforcing the notion that septins play a relevant role in morphogenesis.

Septin localization and dynamics in C. albicans yeast cells is indistinguishable from the pattern described for S. cerevisiae, and the septin rings formed at the bud neck have cell-cycle regulated frozen and fluid states. However, hyphal induction results in important differences in these aspects of septin biology [84-86]. As mentioned above, the localization pattern varies and, in addition, the dynamic properties of the septin ring are also different. Study of septin dynamics using FRAP analysis has revealed that in hyphal cells septin rings are converted to a "hyphal-specific" state that differs from the frozen and fluid states found in yeast cells, and it is characterized by a highly dynamic state of Cdc10, which is constantly exchanged between the ring and a cytoplasmic pool [85]. This modification of septin ring dynamics is crucial in the regulation of several aspects of hyphal growth, as discussed below.

The generation of true hyphae in C. albicans is a specific morphogenetic program, different from yeast growth, and thus requires precise control and the coordination of several cellular processes that can be achieved through the expression of hypha-specific genes or through he differential regulation of components that are present constitutively. Septins are necessary for the proper development of true hypha, but they are expressed constitutively. In contrast, it has been shown that they are subject to both hypha-specific modifications and 
regulation by hyphal determinants; these modifications are essential for the development and maintenance of hyphal growth, and also for the inhibition of cell separation following cytokinesis that is intrinsic to hyphal development $[85,95]$.

The conversion of yeast septin rings to the "hyphal-specific" state is an example of the differential regulation of components that are present in both yeast and hyphal cells, since it is dependent on the presence of Shs1/Sep7 and on its phosphorylation by the hypha-specific cyclin-CDK complex Hgc1-Cdc28 (Figure 3). An important question is whether this modification has any biological relevance to hyphal development. Interestingly, it has been shown that it is of key importance for cell separation of the hyphal compartments to be inhibited, thus allowing them to remain attached to form true hyphae. sep $7 \Delta$ mutants are able to form normal hyphae upon induction, but the different compartments separate after cytokinesis (Figure 2), indicating that Sep7 is essential for separation to be inhibited during hyphal growth. In C. albicans, activation of the cell separation program after cytokinesis requires the Cdc14 phosphatase. It has been shown that this phosphatase localizes to the septum of yeast cells during cytokinesis but not to the hyphal septum, and it was proposed that this localization at the bud neck would be essential to activate the cell separation program regulated by the transcription factor Ace2 [96]. In sep $7 \Delta$ mutants, septin-ring dynamics in hyphae is the same as in yeasts; that is, no exchange of Cdc10 with the cytoplasm occurs, and Cdc14 can localize to the hyphal septum. This in turn activates the Ace2-dependent cell separation program, and mutant hyphae separate by controlled degradation of the septum, mediated by hydrolytic enzymes such as the chitinase Cht3 and the endo-glucanase Eng1 [96-99] (Figure 3). Thus, the reason for the exclusion of the Cdc14 phosphatase from the septum region of hyphal cells could be a change in the scaffolding properties of the "hyphal-specific" septin rings mediated by Sep7. In support of this model, it has been shown that the separation phenotype of sep $7 \Delta$ mutants is abolished when $C D C 14$ is deleted, indicating that the separation of hyphal compartments is due the activation of the separation program [85].

The modification of the dynamic properties and function of the septin ring during a developmental program to adapt it to specific requirements highlights the importance of post- 
translational modifications of specific subunits in the fine-tuned regulation of septin ring properties, and suggests that Sep7 might be an important regulatory subunit of septins, at least during hyphal growth. Sep7 is not important for the assembly of the septin complexes or for the integrity of septin filaments, since $\operatorname{sep} 7 \Delta$ mutants have little or no morphological defects (other than the above-mentioned separation of hyphal compartments), suggesting that the other septins can assemble in vivo and polymerize into filaments in its absence. However, Sep 7 seems to integrate different signals during the hyphal developmental program that are necessary to modify the dynamic properties of the septin rings. Indeed, Sep7 is the largest of the septins and contains two regions with a high content in Ser/Thr (one between the septin element and the coiled-coil domain and another at the C-terminus after the coiled-coil) that may be the target of multiple phosphorylation events by different kinases. Indeed, Sep7 is phosphorylated in an Hgc1/Cdk1-dependent manner during hyphal growth. At the same time, these results raise several interesting questions that could be the subject for future research. One of them is how Sep7 can be assembled into the structure of the septin filaments. Assuming that the arrangement of the monomers in C. albicans septin filaments is similar to the proposed structure of the $S$. cerevisiae heterooctamer $[49,50]$, a key question is whether in vivo Sep7 is also able to polymerize into the main filament or whether it binds the septin filament in a lateral position, perhaps by interaction through the coiled-coil domains. The answer to this question could be of great relevance to understand how Sep7 regulates the dynamics of septin rings. Since the structure of $S$. cerevisiae septin complexes was determined in the absence of Shs1/Sep7, it will be very interesting to check whether the polymerization properties of purified septins is altered in the presence of this regulatory subunit. Another important question is how Cdc10 may exhibit very dynamic behaviour during hyphal growth, constantly exchanging with the cytoplasmic pool, without affecting the structure of the septin filaments. One possibility is that the basic polymerization unit of $C$. albicans septin subunits during hyphal growth would be different to that found in yeast cells (the S. cerevisiae heterooctamer), this change being regulated by hyphaspecific signals. Indeed, our unpublished observations indicate that Cdc11 shows behaviour similar to that of Cdc10 (A. Gonzalez-Novo, unpublished observations), suggesting that Cdc3- 
Cdc12 could form the static core of hyphal septin filaments, while Cdc10-Cdc11 freely exchange with the cytoplasmic pool. Interestingly, in $S$. cerevisiae filament pairing between adjacent filaments is mediated by the coiled-coil regions of Cdc3 and Cdc12 [49]. Alternatively, it is possible that the structure of the basic polymerization unit might be the same in yeast and hyphal cells and that during hyphal growth additional monomers of the dynamic subunits $(\mathrm{Cdc} 10-\mathrm{Cdc} 11)$ are able to bind and "decorate" the septin filaments in a Sep7-dependent manner, thus modifying the scaffolding properties of the septin rings. Clearly, the answer to this question would be of great interest for an understanding of the structure-function relationship of septins, and will merit special attention in the future. The last, but not least, important question is whether this "hyphal-specific" state is exclusive to C. albicans or whether it is also present in other fungi. It will undoubtedly be very interesting to analyze septin dynamics in other fungi, especially filamentous fungi in which cell separation does not occur, to test whether this a specific mechanism that $C$. albicans has developed to adapt to the specific requirements imposed by the ability to switch between different morphologies or whether it is a common feature of filamentous growth.

Challenging C. albicans yeasts with hyphal inducers results in very fast responses, which suggests that post-transcriptional modifications of pre-existing proteins play an important role in this morphological transition. Recently, in a very convincing set of experiments Shina and coworkers provided strong biochemical and cellular evidence that one of the proteins that is modified during hyphal development is Cdc11 [95]. Phosphorylation of a serine residue in the $\mathrm{C}$ terminus of Cdc11 (Ser395) by the kinase Gin4 in the previous yeast cell cycle is the priming signal for the phosphorylation of a contiguous serine (Ser394) by the Cdc28/Ccn1 complex in stimulated cells (Cdc11 phosphorylation by $\operatorname{Ccn} 1-\mathrm{Cdc} 28$ can be detected five minutes after hyphal induction). This double phosphorylation is essential for the induction and maintenance of hyphal growth (Figure 4) since a CCN1 deletion or a Ser394 mutation in Cdc11 results in swollen hyphal tips after the first septum has been assembled (Figure 2). The phenotype of a $\operatorname{ccn} 1 \Delta$ mutant was suppressed by deletion of $C D C 11$ or by the generation of double phosphomimetic mutations on Cdc11. Gin4 phosphorylates Cdc11 in the cell cycle prior to 
hyphal induction, thus generating cells that can respond quickly if hyphal-inducing signals are received. This implies the existence of a mechanistic relationship between the cell cycle and septins, which the authors suggested could be a conserved pathway for polarized morphogenesis. Interestingly, it has recently been shown that another S. cerevisiae septin, Shs1, is also primed by phosphorylation regulated by G1 cyclin-dependent kinases, and that this phosphorylation is necessary for Gin 4 recruitment to the septin ring during mitosis and, as a consequence, for the regulation of later cell cycle events [100].

One intriguing result of this work is that Cdc11 phosphorylation at early times after hyphal induction results in morphological defects at later times in hypha development; after the septin ring has been formed. In many organisms, polarized growth is dependent on the polarized secretion of vesicles to the growing region of the cell to provide new cell wall and membrane material. This process is dependent on a multi-protein complex called the exocyst. Work from $S$. cerevisiae has shown that upon bud emergence septins direct cell growth to the base of the bud to produce the typical swelling on the daughter side of the neck [101]. In C. albicans hyphae, the lateral walls are parallel and lack any constrictions or swellings after the septum, indicating that hyphal growth requires polarized secretion to the hyphal septin ring to be suppressed and targeted to the filament tip (Figure 2). Interestingly, a $C$. albicans $\sec 3 \Delta$ mutant -which encodes an exocyst subunit- has a phenotype similar to that of the $\operatorname{ccn} 1 \Delta$ mutant or the Cdc11-S394A mutant -that is, swollen tips after the septin ring has been assembled (Figure 2)-, indicating that the exocyst and the septin complexes interact in this process. Moreover, deletion of either $C D C 10$ or $C D C 11$ in a $\sec 3 \Delta$ mutant restores proper hyphal growth [102], and a physical interaction between septin and exocyst components has been demonstrated in coimmunoprecipitation assays, indicating that both genetic and physical interactions between these two complexes are essential for proper hyphal growth. An interesting question is which molecular mechanisms are involved in the inhibition of secretion to the septin ring during hyphal growth. The above results provide several interesting possibilities. It is possible that phosphorylation by Gin 4 and $\mathrm{Cdc} 28 / \mathrm{Ccn} 1$ may reduce the affinity of $\mathrm{Cdc} 11$ for the exocyst subunit Sec3, thus targeting secretion predominantly to the tip [103]. The conversion of the 
septin rings to the "hyphal-specific" state and the modification of its scaffolding properties (as discussed above for Cdc14) might be an alternative, or perhaps an additional, mechanism to target secretion to the growing tip. If this were the case, it would suggest that septin subunits at the different structures of the hyphae might have different modifications or modes of regulation to accomplish specific functions. Unfortunately, Cdc10 dynamics has not been analyzed in the basal band or the growing tip in answer this question, but this could be an open field for research in the near future.

All the above results suggest that upon hyphal induction, septins play key roles in establishing and maintaining the morphogenetic traits that are specific to this growth pattern, serving as positive signals for polarized secretion and growth at the tip of germ tubes $[95,102]$ and acting as inhibitory regions for cell separation [85]. However, our understanding of the signals and factors that regulate septin structure, assembly and localization is still sparse. The co-localization of septins with lipid rafts in hyphal cells or with the integrin-like protein Int 1 in both yeast and hyphal cells, together with the fact that expression of Int1 in $S$. cerevisiae strongly alters septin localization, provides some clues $[104,105]$. One factor that is known to regulate septin assembly is the kinase Gin4. When this kinase is placed under the control of the MET3 promoter and its expression is blocked, C. albicans cells can undergo the yeast-to-hypha transition perfectly, but the septin rings cannot be assembled [106]; this suggests an essential role of this kinase in septin ring assembly, as in $S$. cerevisiae $[33,37,39,69]$. Curiously, the assembly of the septin basal band at the base of the germ tube is not dependent on the presence of this kinase [106], indicating that C. albicans septins are subjected to differential signals that control their assembly, depending on the morphogenetic state and even on the kind of structure. Recently, it has been shown that the Cdc42 GTPase-activating proteins (GAPs) Bem3 and Rga2 would have some redundant role in the regulation of septin ring localization, although the exact contribution of these proteins is unclear [107].

Interestingly, although septins are highly conserved between $S$. cerevisiae and $C$. albicans, septin modifications are different in these two fungi. The important serine residues that are phosphorylated in C. albicans Cdc11 are not conserved in its $S$. cerevisiae orthologue 
[95] and, conversely, $\mathrm{ScCdc} 3$ has two phosphorylatable residues at its $\mathrm{C}$ terminus that are important for the stability of the septin ring [41] and that are not conserved in its C. albicans orthologue, suggesting that these organisms have developed different and specific systems for the regulation of septin dynamics and functions. In addition, septin dynamics during hyphal growth is completely different from that seen in S. cerevisiae, indicating that specific morphogenetic programs have specific septin regulation pathways [85]. Finally, in contrast to what happens in S. cerevisiae [40], in C. albicans septins are not modified by SUMO [108], although some of the septin-interacting proteins are SUMO-modified proteins. Interestingly, these modified proteins mainly localize to the mother cell, similarly to $S$. cerevisiae. Further investigations in this human fungal pathogen addressing septin assembly regulatory signals, the specific modifications of septins in hyphal cells and their role in polarization should add important information to our knowledge of these proteins and the processes they are involved in.

\section{Ashbya gossypii}

The extension of morphogenesis studies to other yeasts and filamentous fungi is currently providing additional insight into the functions of septins. Ashbya gossypii is a filamentous fungus closely related to $S$. cerevisiae, but one that grows exclusively in a filamentous fashion, generating long multinucleated hyphae. After germination of the haploid spore, two germ tubes are formed on opposing sites, in which lateral branches are developed. Hyphae are compartmentalized by septa, and one compartment typically contains around eight nuclei (for a review on Ashbya biology, see [109]). Ashbya contains six septin genes in its genome, five of which are orthologues to the mitotic budding yeast septins plus a duplication of CDC11. Surprisingly, in A. gossypii septins are not essential, presumably because cytokinesis itself is not essential $[110,111]$, but they are required for the selection and organization of the septation site, to promote mitosis, and for asexual spores to be formed [110-112]. Owing to its growth pattern, in this organism the septins assemble into morphologically distinct classes of structures that vary in dimensions, intensities and positions within the filament. Thus, septin structures are 
present at the growing tips of the hyphae as a diffuse structure composed of short filaments arranged parallel to the long axis and also at the branching points. As the filament grows, septin structures are also found at constant distances in the region between the growing tip and the spore, which have been named inter-region rings. The inter-region rings persist for variable times before splitting in two adjacent rings that mark the septation point. Finally, septins also congregate at the cap of the emerging branches $[110,113]$. In spite of the different organization, all septins are present in all classes of structures, which raises the question of how they are assembled and regulated. Interestingly, septins have different dependence on regulatory proteins since the formation of inter-region structures requires the activity of the AgGin4 and AgElm1 kinases, while those at the branches and tip do not [113]. What is also interesting is that the dependence on AgGin4 for assembly of the inter-region rings and septation sites is similar to that described above for C. albicans, suggesting that this kinase might play a similar role in the regulation of specific septin structures during hyphal growth in both yeasts. Based on the results obtained during $C$. albicans hyphal growth, it will be very interesting to analyze the dynamics of the septin subunits inside each of these structures to test whether it varies from one to the other. Another factor that controls the reorganization of septin structures is the $\mathrm{PCH}$ (pombe

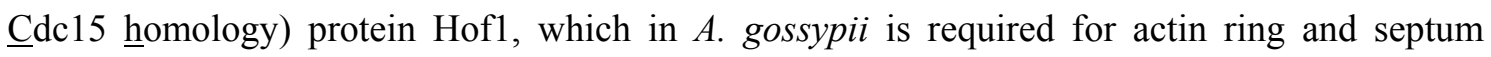
formation. In addition, Hofl is required for the reorganization of Sep7 bars parallel to the growing axis towards rings in mature hyphae [111].

A. gossypii is becoming a new and interesting model system to study septin function and regulation, since septins are not essential and because septin assembly, maturation, and organization do not require the nuclear cycle, providing an easily manageable system for studying cell cycle-independent regulation of septins [113]. Moreover, in A. gossypii septins are required to generate the appropriate environment for mitosis, and hence this event is mainly found in the vicinity of septin structures, although not all septin structures function in the same way: mitosis is more frequent at branching septin structures than at growing-tip septin structures, suggesting that the different organization is perhaps accompanied by different functions [110]. This idea is supported by the observation that septin mutants have a reduced 
frequency of mitosis near the branches but not at the tips. This is very interesting, since in other fungi such as $C$. albicans several septin structures can be found, and it is therefore possible that these structures could be performing different and independent functions, warranting further investigations, as discussed above for secretion. Furthermore, it has been shown that during $C$. albicans hyphal growth, the nucleus migrates to the vicinity of the presumptive septum site (where the septin ring is assembled) before its division [114]. In Aspergillus nidulans, septin ring assembly at the base of the branches is the signal required for the nucleus to re-enter mitosis, but the exact nature of this relationship remains unknown [115]. In addition to this, the septin ring in budding yeast determines the position of mitosis, and this also occurs in $C$. albicans yeast cells. However, it seems that in C. albicans hyphae septins are not the only factor required to position the mitosis plane since in gin $4 \Delta$ cells, which lack septin rings in the germ tube, mitosis apparently takes place normally [106]. Taken together, these results suggest that septins are involved in the control of nuclear division, although the exact process remains to be clearly elucidated. A clue that can help to answer this question is the observation that in $A$. gosypii mitosis localization is not only dependent on septins, but also on AgSwe1, the orthologue to the S. cerevisiae kinase Swe1 [110]. The authors proposed that perhaps an adaptation of the $S$. cerevisiae morphogenetic checkpoint could be involved in the regulation of mitosis position: recruitment of AgSwe1 to the septin rings would result in a local depletion or inhibition of this kinase, this allowing the activation of mitosis near the rings.

\section{Aspergillus nidulans}

In A. nidulans, septins are also related to septation, branching, and conidiophore formation [115]. Interestingly, in this case septin rings are disassembled as the filament grows, persisting only at the apical-most structure. This suggests that in this organism septins could exert apical polarization functions. At this juncture, it is important to recall that in the polymorphic fungus $C$. albicans the septin rings of the hyphae are not disassembled immediately after septum assembly, but persist as the filament grows. In this case, septins may function as inhibitory markers for cell separation, although they could also be polarity 
landmarks, because branching always takes place in the vicinity of old septin rings. It is also noteworthy that in $C$. albicans hyphae septins can be found as a cap at the tip of the germ tube. Thus, it is tempting to speculate that apical septin structures could function as scaffolds required for polarized growth and secretion, while the septin rings could maintain the integrity of the filaments by repressing cell separation and, at the same time, determine landmarks for branching. All these aspects of septins related to polarity and branching should be further investigated to unequivocally clarify this issue of crucial importance.

\section{Other yeast and fungi}

Another economically important fungus is the phytopathogen Ustilago maydis, which contains four septin genes in its genome. To date, only sep 3 -the orthologue of the S. cerevisiae Cdc11- has been thoroughly analyzed. sep3 $\Delta$ strains have different morphological defects, including swollen and misshapen cells that often grow as clusters of cells, multinucleated cells, or with inappropriately positioned or multiple septa [116], indicating that this septin is necessary for normal morphogenesis and cytokinesis. In addition, the dimorphic transition from the haploid budding yeast form to the dikaryotic filamentous growth, which is crucial for pathogenic development in maize, is defective. More recently, it has been reported that deletion of sep1 -the orthologue of the S. cerevisiae Cdc3- also produces morphological defects consistent with the inability to undergo proper polarized cell growth in $U$. maydis yeast cells [117].

In the fission yeast Schizosaccharomyces pombe, none of the four mitotic septins -Spn1, Spn2, Spn3 and Spn4- is essential, and deletion mutants only display mild morphogenetic defects. The only remarkable phenotype associated with the absence of septins is a delay in septum degradation after cytokinesis, which results in a characteristic chained phenotype [118, 119]. The only function described so far for fission yeast septins is to serve as positional markers to target the secretory vesicles loaded with the hydrolytic enzymes required for the controlled dissolution of the septum (i.e., the $\alpha$-glucanase Agn1 and the $\beta$-glucanase Eng1 [120, 121]) to the septum area in an exocyst-dependent manner [122]. The same phenotype is 
observed in cells that lack the anillin homologue Mid2, which is required for proper septin ring organization in this yeast: in mid $2 \Delta$ mutant cells, the septin rings are not stable, and the septins diffuse to the septum region, forming discs instead of rings [118, 119]. Interestingly, the transcription factor Ace2 not only regulates the expression of genes required for cell separation, but also controls septin ring stability through the expression of mid $2^{+}$[123]. These results suggest that a correct septin ring is essential for the polarized secretion of hydrolytic enzymes in S. pombe.

\section{CONCLUSIONS}

Septins are conserved between different fungi, both in sequence and in function, although they show peculiarities and differences in the functions that underlie the nature of each organism. In addition to the species described in this review, septins are also present in many other fungi [27], and detailed studies may uncover new and interesting functions of these proteins specifically adapted to the biology of each organism. This conservation is spread throughout evolution to higher eukaryotes, in which septins also have the same basic functions, involving cytokinesis, scaffolding or polarity regulation. Nevertheless, the better knowledge of septin biology gained in recent years has shown that septins have some specific functions, and they have even been related to tumorogenesis [11] and neurodegenerative diseases, such as Alzheimer's, Parkinson's or hereditary neuralgic amyotrophy [124-126]. The use of fungi as model organisms has proved to be of enormous relevance and interest, and should also be of huge importance in future years in our quest to reveal surprising and unexpected functions in these organisms.

\section{ACKNOWLEDGMENTS}

We thank Jaime Correa for critical reading of the manuscript and Nick Skinner for language revision. This work was supported by grants from Junta de Castilla y Léon SA013B05 (to JJ) and Ministerio de Ciencia y Tecnología BFU2007-60390 (to CRV). 


\section{REFERENCES}

[1] Hartwell L.H., Genetic control of the cell division cycle in yeast. IV. Genes controlling bud emergence and cytokinesis, Exp. Cell Res., 1971, 69, 265-276

[2] Carroll C.W., Altman R., Schieltz D., Yates J.R., Kellogg D., The septins are required for the mitosis-specific activation of the Gin4 kinase, J. Cell Biol., 1998, 143, 709-717

[3] Mino A., Tanaka K., Kamei T., Umikawa M., Fujiwara T., Takai Y., Shs1p: a novel member of septin that interacts with Spa2p, involved in polarized growth in Saccharomyces cerevisiae, Biochem. Biophys. Res. Commun., 1998, 251, 732-736

[4] De Virgilio C., DeMarini D.J., Pringle J.R., SPR28, a sixth member of the septin gene family in Saccharomyces cerevisiae that is expressed specifically in sporulating cells, Microbiology, 1996, 142, 2897-2905

[5] Fares H., Goetsch L., Pringle J.R., Identification of a developmentally regulated septin and involvement of the septins in spore formation in Saccharomyces cerevisiae, J. Cell Biol., 1996, 132, 399-411

[6] Longtine M.S., Bi E., Regulation of septin organization and function in yeast, Trends Cell. Biol., 2003, 13, 403-409

[7] Longtine M.S., DeMarini D.J., Valencik M.L., Al-Awar O.S., Fares H., De Virgilio C., et al., The septins: roles in cytokinesis and other processes, Curr. Opin. Cell Biol., 1996, 8, 106-119

[8] Faty M., Fink M., Barral Y., Septins: a ring to part mother and daughter, Curr. Genet., 2002, $41,123-131$

[9] Silverman-Gavrila R.V., Silverman-Gavrila L.B., Septins: new microtubule interacting partners, ScientificWorldJournal, 2008, 8, 611-620

[10] Spiliotis E.T., Nelson W.J., Here come the septins: novel polymers that coordinate intracellular functions and organization, J. Cell Sci., 2006, 119, 4-10

[11] Hall P.A., Russell S.E., The pathobiology of the septin gene family, J. Pathol., 2004, 204, 489-505

[12] Kinoshita M., Assembly of mammalian septins, J. Biochem., 2003, 134, 491-496

[13] Kinoshita M., Diversity of septin scaffolds, Curr. Opin. Cell Biol., 2006, 18, 54-60

[14] Martinez C., Ware J., Mammalian septin function in hemostasis and beyond, Exp. Biol. Med., 2004, 229, 1111-1119

[15] Douglas L.M., Alvarez F.J., McCreary C., Konopka J.B., Septin function in yeast model systems and pathogenic fungi, Eukaryot. Cell, 2005, 4, 1503-1512

[16] Finger F.P., One ring to bind them. Septins and actin assembly, Dev. Cell., 2002, 3, 761763

[17] Gladfelter A.S., Pringle J.R., Lew D.J., The septin cortex at the yeast mother-bud neck, Curr. Opin. Microbiol, 2001, 4, 681-689 
[18] Kinoshita M., The septins, Genome Biol., 2003, 4, 236

[19] Versele M., Thorner J., Some assembly required: yeast septins provide the instruction manual, Trends Cell. Biol., 2005, 15, 414-424

[20] Caudron F., Barral Y., Septins and the lateral compartmentalization of eukaryotic membranes, Dev. Cell., 2009, 16, 493-506

[21] Gladfelter A.S., Sudbery P. (2008) Septins in four model fungal systems: diversity in form and function. In The Septins (Hall P.A. Hilary Russell S.E. and Pringle J.R., eds.), pp. 125146, John Wiley \& Sons

[22] Field C.M., Kellogg D., Septins: cytoskeletal polymers or signalling GTPases?, Trends Cell. Biol., 1999, 9, 387-394

[23] Versele M., Gullbrand B., Shulewitz M.J., Cid V.J., Bahmanyar S., Chen R.E., et al., Protein-protein interactions governing septin heteropentamer assembly and septin filament organization in Saccharomyces cerevisiae, Mol. Biol. Cell, 2004, 15, 4568-4583

[24] Casamayor A., Snyder M., Molecular dissection of a yeast septin: distinct domains are required for septin interaction, localization, and function, Mol. Cell. Biol., 2003, 23, 27622777

[25] Zhang J., Kong C., Xie H., McPherson P.S., Grinstein S., Trimble W.S., Phosphatidylinositol polyphosphate binding to the mammalian septin $\mathrm{H} 5$ is modulated by GTP, Curr. Biol., 1999, 9, 1458-1467

[26] An H., Morrell J.L., Jennings J.L., Link A.J., Gould K.L., Requirements of fission yeast septins for complex formation, localization, and function, Mol. Biol. Cell, 2004, 15, 55515564

[27] Pan F., Malmberg R.L., Momany M., Analysis of septins across kingdoms reveals orthology and new motifs, BMC Evol. Biol., 2007, 7, 103

[28] Byers B., Goetsch L., A highly ordered ring of membrane-associated filaments in budding yeast, J. Cell Biol., 1976, 69, 717-721.

[29] Cid V.J., Adamikova L., Sanchez M., Molina M., Nombela C., Cell cycle control of septin ring dynamics in the budding yeast, Microbiology, 2001, 147, 1437-1450.

[30] Gladfelter A.S., Moskow J.J., Zyla T.R., Lew D.J., Isolation and characterization of effector-loop mutants of CDC42 in yeast, Mol. Biol. Cell, 2001, 12, 1239-1255.

[31] Iwase M., Luo J., Nagaraj S., Longtine M., Kim H.B., Haarer B.K., et al., Role of a Cdc42p effector pathway in recruitment of the yeast septins to the presumptive bud site, Mol. Biol. Cell, 2006, 17, 1110-1125

[32] Longtine M.S., Theesfeld C.L., McMillan J.N., Weaver E., Pringle J.R., Lew D.J., Septindependent assembly of a cell cycle-regulatory module in Saccharomyces cerevisiae, Mol. Cell. Biol., 2000, 20, 4049-4061.

[33] Mortensen E.M., McDonald H., Yates J., 3rd, Kellogg D.R., Cell cycle-dependent 
assembly of a Gin4-septin complex, Mol. Biol. Cell, 2002, 13, 2091-2105

[34] Versele M., Thorner J., Septin collar formation in budding yeast requires GTP binding and direct phosphorylation by the PAK, Cla4, J. Cell Biol., 2004, 164, 701-715

[35] Bouquin N., Barral Y., Courbeyrette R., Blondel M., Snyder M., Mann C., Regulation of cytokinesis by the Elm1 protein kinase in Saccharomyces cerevisiae, J. Cell Sci., 2000, $113,1435-1445$

[36] Cvrckova F., De Virgilio C., Manser E., Pringle J.R., Nasmyth K., Ste20-like protein kinases are required for normal localization of cell growth and for cytokinesis in budding yeast, Genes Dev., 1995, 9, 1817-1830

[37] Longtine M.S., Fares H., Pringle J.R., Role of the yeast Gin4p protein kinase in septin assembly and the relationship between septin assembly and septin function, J. Cell Biol., $1998,143,719-736$

[38] Weiss E.L., Bishop A.C., Shokat K.M., Drubin D.G., Chemical genetic analysis of the budding-yeast p21-activated kinase Cla4p, Nat. Cell. Biol., 2000, 2, 677-685

[39] Dobbelaere J., Gentry M.S., Hallberg R.L., Barral Y., Phosphorylation-dependent regulation of septin dynamics during the cell cycle, Dev. Cell., 2003, 4, 345-357

[40] Johnson E.S., Blobel G., Cell cycle-regulated attachment of the ubiquitin-related protein SUMO to the yeast septins, J. Cell Biol., 1999, 147, 981-994

[41] Tang C.S., Reed S.I., Phosphorylation of the septin Cdc3 in G1 by the Cdc28 kinase is essential for efficient septin ring disassembly, Cell Cycle, 2002, 1, 42-49.

[42] Pablo-Hernando M.E., Arnáiz-Pita Y., Tachikawa H., Rey F.del, Neiman A.M., Vázquez de Aldana C.R., Septins localize to microtubules during nutritional limitation in Saccharomyces cerevisiae, BMC Cell. Biol., 2008, 9, 55

[43] McMurray M.A., Thorner J., Septin stability and recycling during dynamic structural transitions in cell division and development, Curr. Biol., 2008, 18, 1203-1208

[44] McMurray M.A., Thorner J., Reuse, replace, recycle. Specificity in subunit inheritance and assembly of higher-order septin structures during mitotic and meiotic division in budding yeast, Cell Cycle, 2009, 8, 195-203

[45] Field C.M., al-Awar O., Rosenblatt J., Wong M.L., Alberts B., Mitchison T.J., A purified Drosophila septin complex forms filaments and exhibits GTPase activity, J. Cell Biol., 1996, 133, 605-616

[46] Frazier J.A., Wong M.L., Longtine M.S., Pringle J.R., Mann M., Mitchison T.J., et al., Polymerization of purified yeast septins: evidence that organized filament arrays may not be required for septin function, J. Cell Biol., 1998, 143, 737-749

[47] Kinoshita M., Field C.M., Coughlin M.L., Straight A.F., Mitchison T.J., Self- and actintemplated assembly of mammalian septins, Dev. Cell., 2002, 3, 791-802

[48] Mendoza M., Hyman A.A., Glotzer M., GTP binding induces filament assembly of a 
recombinant septin, Curr. Biol., 2002, 12, 1858-1863

[49] Bertin A., McMurray M.A., Grob P., Park S.S., Garcia G., 3rd, Patanwala I., et al., Saccharomyces cerevisiae septins: supramolecular organization of heterooligomers and the mechanism of filament assembly, Proc. Natl. Acad. Sci. USA, 2008, 105, 8274-8279

[50] Sirajuddin M., Farkasovsky M., Hauer F., Kuhlmann D., Macara I.G., Weyand M., et al., Structural insight into filament formation by mammalian septins, Nature, 2007, 449, 311315

[51] John C.M., Hite R.K., Weirich C.S., Fitzgerald D.J., Jawhari H., Faty M., et al., The Caenorhabditis elegans septin complex is nonpolar, EMBO J., 2007, 26, 3296-3307

[52] Barral Y., Kinoshita M., Structural insights shed light onto septin assemblies and function, Curr. Opin. Cell Biol., 2008, 20, 12-18

[53] Weirich C.S., Erzberger J.P., Barral Y., The septin family of GTPases: architecture and dynamics, Nat. Rev. Mol. Cell. Biol., 2008, 9, 478-489

[54] Nagaraj S., Rajendran A., Jackson C.E., Longtine M.S., Role of nucleotide binding in septin-septin interactions and septin localization in Saccharomyces cerevisiae, Mol. Cell. Biol., 2008, 28, 5120-5137

[55] Iwase M., Luo J., Bi E., Toh-e A., Shs1 plays separable roles in septin organization and cytokinesis in Saccharomyces cerevisiae, Genetics, 2007, 177, 215-229

[56] Caviston J.P., Longtine M., Pringle J.R., Bi E., The role of Cdc42p GTPase-activating proteins in assembly of the septin ring in yeast, Mol. Biol. Cell, 2003, 14, 4051-4066

[57] Vrabioiu A.M., Mitchison T.J., Structural insights into yeast septin organization from polarized fluorescence microscopy, Nature, 2006, 443, 466-469

[58] Keaton M.A., Lew D.J., Eavesdropping on the cytoskeleton: progress and controversy in the yeast morphogenesis checkpoint, Curr. Opin. Microbiol, 2006, 9, 540-546

[59] Douglas L.M., Alvarez F.J., McCreary C., Konopka J.B., Septin function in yeast model systems and pathogenic fungi, Eukaryot. Cell, 2005, 4, 1503-1512

[60] Chant J., Generation of cell polarity in yeast, Curr. Opin. Cell Biol., 1996, 8, 557-565

[61] Ford S.K., Pringle J.R., Cellular morphogenesis in the Saccharomyces cerevisiae cell cycle: localization of the CDC11 gene product and the timing of events at the budding site, Dev. Genet., 1991, 12, 281-292

[62] Halme A., Michelitch M., Mitchell E.L., Chant J., Bud10p directs axial cell polarization in budding yeast and resembles a transmembrane receptor, Curr. Biol., 1996, 6, 570-579

[63] Kang P.J., Sanson A., Lee B., Park H.O., A GDP/GTP exchange factor involved in linking a spatial landmark to cell polarity, Science, 2001, 292, 1376-1378

[64] Roemer T., Vallier L.G., Snyder M., Selection of polarized growth sites in yeast, Trends Cell. Biol., 1996, 6, 434-441

[65] Sanders S.L., Herskowitz I., The BUD4 protein of yeast, required for axial budding, is 
localized to the mother/BUD neck in a cell cycle-dependent manner, J. Cell Biol., 1996, $134,413-427$

[66] Castillon G.A., Adames N.R., Rosello C.H., Seidel H.S., Longtine M.S., Cooper J.A., et al., Septins have a dual role in controlling mitotic exit in budding yeast, Curr. Biol., 2003, $13,654-658$

[67] Grava S., Schaerer F., Faty M., Philippsen P., Barral Y., Asymmetric recruitment of dynein to spindle poles and microtubules promotes proper spindle orientation in yeast, Dev. Cell., 2006, 10, 425-439

[68] Kusch J., Meyer A., Snyder M.P., Barral Y., Microtubule capture by the cleavage apparatus is required for proper spindle positioning in yeast, Genes Dev., 2002, 16, 1627-1639

[69] Barral Y., Parra M., Bidlingmaier S., Snyder M., Nim1-related kinases coordinate cell cycle progression with the organization of the peripheral cytoskeleton in yeast, Genes Dev., 1999, 13, 176-187

[70] Hanrahan J., Snyder M., Cytoskeletal activation of a checkpoint kinase, Mol. Cell, 2003, $12,663-673$

[71] Lew D.J., The morphogenesis checkpoint: how yeast cells watch their figures, Curr. Opin. Cell Biol., 2003, 15, 648-653

[72] Cid V.J., Shulewitz M.J., McDonald K.L., Thorner J., Dynamic localization of the Swe1 regulator Hsl7 during the Saccharomyces cerevisiae cell cycle, Mol. Biol. Cell, 2001, 12, 1645-1669

[73] Shulewitz M.J., Inouye C.J., Thorner J., Hsl7 localizes to a septin ring and serves as an adapter in a regulatory pathway that relieves tyrosine phosphorylation of $\mathrm{Cdc} 28$ protein kinase in Saccharomyces cerevisiae, Mol. Cell. Biol., 1999, 19, 7123-7137

[74] Moffat J., Andrews B., Ac'septin' a signal: kinase regulation by septins, Dev. Cell., 2003, 5, 528-530

[75] Kozubowski L., Larson J.R., Tatchell K., Role of the septin ring in the asymmetric localization of proteins at the mother-bud neck in Saccharomyces cerevisiae, Mol. Biol. Cell, 2005, 16, 3455-3466

[76] Vrabioiu A.M., Mitchison T.J., Symmetry of septin hourglass and ring structures, J. Mol. Biol., 2007, 372, 37-49

[77] Barral Y., Mermall V., Mooseker M.S., Snyder M., Compartmentalization of the cell cortex by septins is required for maintenance of cell polarity in yeast, Mol. Cell, 2000, 5, $841-851$

[78] Seshan A., Bardin A.J., Amon A., Control of Lte1 localization by cell polarity determinants and Cdc14, Curr. Biol., 2002, 12, 2098-2110

[79] Takizawa P.A., DeRisi J.L., Wilhelm J.E., Vale R.D., Plasma membrane compartmentalization in yeast by messenger RNA transport and a septin diffusion barrier, 
Science, 2000, 290, 341-344.

[80] Dobbelaere J., Barral Y., Spatial coordination of cytokinetic events by compartmentalization of the cell cortex, Science, 2004, 305, 393-396

[81] Luedeke C., Frei S.B., Sbalzarini I., Schwarz H., Spang A., Barral Y., Septin-dependent compartmentalization of the endoplasmic reticulum during yeast polarized growth, J. Cell Biol., 2005, 169, 897-908

[82] Shcheprova Z., Baldi S., Frei S.B., Gonnet G., Barral Y., A mechanism for asymmetric segregation of age during yeast budding, Nature, 2008, 454, 728-734

[83] Soll D.R., Mitchell L.H., Filament ring formation in the dimorphic yeast Candida albicans, J. Cell Biol., 1983, 96, 486-493

[84] Warenda A.J., Konopka J.B., Septin function in Candida albicans morphogenesis, Mol. Biol. Cell, 2002, 13, 2732-2746

[85] González-Novo A., Correa-Bordes J., Labrador L., Sánchez M., Vázquez de Aldana C.R., Jiménez J., Sep7 is essential to modify septin ring dynamics and inhibit cell separation during Candida albicans hyphal growth, Mol. Biol. Cell, 2008, 19, 1509-1518

[86] Sudbery P.E., The germ tubes of Candida albicans hyphae and pseudohyphae show different patterns of septin ring localization, Mol. Microbiol., 2001, 41, 19-31

[87] González-Novo A., Jiménez J., García M.J., Ríos-Serrano I., Pla J., Jiménez A., et al., Dynamics of $\mathrm{CaCdc10}$, a septin of Candida albicans, in living cells and during infection, Int. Microbiol., 2004, 7, 105-112

[88] Liu H., Co-regulation of pathogenesis with dimorphism and phenotypic switching in Candida albicans, a commensal and a pathogen, Int. J. Med. Microbiol., 2002, 292, 299311

[89] Lo H.J., Kohler J.R., DiDomenico B., Loebenberg D., Cacciapuoti A., Fink G.R., Nonfilamentous C. albicans mutants are avirulent, Cell, 1997, 90, 939-949

[90] Odds F.C., Candida and candidosis 2nd edn, 1988, Bailliére Tindall, London

[91] Berman J., Sudbery P.E., Candida albicans: A molecular revolution built on lessons from budding yeast, Nat. Rev. Genet., 2002, 3, 918-932.

[92] Sudbery P., Gow N., Berman J., The distinct morphogenic states of Candida albicans, Trends Microbiol., 2004, 12, 317-324

[93] González-Novo A., Labrador L., Jiménez A., Sánchez-Pérez M., Jiménez J., Role of the septin Cdc10 in the virulence of Candida albicans, Microbiol. Immunol., 2006, 50, 499511

[94] Warenda A.J., Kauffman S., Sherrill T.P., Becker J.M., Konopka J.B., Candida albicans septin mutants are defective for invasive growth and virulence, Infect. Immun., 2003, 71, 4045-4051

[95] Sinha I., Wang Y.M., Philp R., Li C.R., Yap W.H., Wang Y., Cyclin-dependent kinases 
control septin phosphorylation in Candida albicans hyphal development, Dev. Cell., 2007, $13,421-432$

[96] Clemente-Blanco A., González-Novo A., Machín F., Caballero-Lima D., Aragón L., Sánchez M., et al., The Cdc14p phosphatase affects late cell-cycle events and morphogenesis in Candida albicans, J. Cell Sci., 2006, 119, 1130-1143

[97] Dunkler A., Walther A., Specht C.A., Wendland J., Candida albicans CHT3 encodes the functional homolog of the Cts1 chitinase of Saccharomyces cerevisiae, Fungal Genet. Biol., 2005, 42, 935-947

[98] Esteban P.F., Ríos I., García R., Dueñas E., Plá J., Sánchez M., et al., Characterization of the CaENG1 gene encoding an endo-1,3- $\beta$-glucanase involved in cell separation in Candida albicans, Curr. Microbiol., 2005, 51, 385-392

[99] Kelly M.T., MacCallum D.M., Clancy S.D., Odds F.C., Brown A.J., Butler G., The Candida albicans CaACE2 gene affects morphogenesis, adherence and virulence, Mol. Microbiol., 2004, 53, 969-983

[100] Egelhofer T.A., Villen J., McCusker D., Gygi S.P., Kellogg D.R., The septins function in G1 pathways that influence the pattern of cell growth in budding yeast, PLoS ONE, 2008, 3, e2022

[101] Gladfelter A.S., Kozubowski L., Zyla T.R., Lew D.J., Interplay between septin organization, cell cycle and cell shape in yeast, J. Cell Sci., 2005, 118, 1617-1628

[102] Li C.R., Lee R.T., Wang Y.M., Zheng X.D., Wang Y., Candida albicans hyphal morphogenesis occurs in Sec3p-independent and Sec3p-dependent phases separated by septin ring formation, J. Cell Sci., 2007, 120, 1898-1907

[103] Sudbery P., Morphogenesis of a human fungal pathogen requires septin phosphorylation, Dev. Cell., 2007, 13, 315-316

[104] Gale C., Gerami-Nejad M., McClellan M., Vandoninck S., Longtine M.S., Berman J., Candida albicans Intlp interacts with the septin ring in yeast and hyphal cells, Mol. Biol. Cell, 2001, 12, 3538-3549

[105] Martin S.W., Konopka J.B., Lipid raft polarization contributes to hyphal growth in Candida albicans, Eukaryot. Cell, 2004, 3, 675-684

[106] Wightman R., Bates S., Amornrrattanapan P., Sudbery P., In Candida albicans, the Nim1 kinases Gin4 and Hsl1 negatively regulate pseudohypha formation and Gin4 also controls septin organization, J. Cell Biol., 2004, 164, 581-591

[107] Court H., Sudbery P., Regulation of Cdc42 GTPase activity in the formation of hyphae in Candida albicans, Mol. Biol. Cell, 2007, 18, 265-281

[108] Martin S.W., Konopka J.B., SUMO modification of septin-interacting proteins in Candida albicans, J. Biol. Chem., 2004, 279, 40861-40867

[109] Wendland J., Walther A., Ashbya gossypii: a model for fungal developmental biology, 
Nat. Rev. Microbiol., 2005, 3, 421-429

[110] Helfer H., Gladfelter A.S., AgSwe1p regulates mitosis in response to morphogenesis and nutrients in multinucleated Ashbya gossypii cells, Mol. Biol. Cell, 2006, 17, 4494-4512

[111] Kaufmann A., Philippsen P., Of bars and rings: Hofl-dependent cytokinesis in multiseptated hyphae of Ashbya gossypii, Mol. Cell. Biol., 2009, 29, 771-783

[112] Gladfelter A.S., Control of filamentous fungal cell shape by septins and formins, Nat. Rev. Microbiol., 2006, 4, 223-229

[113] DeMay B.S., Meseroll R.A., Occhipinti P., Gladfelter A.S., Regulation of distinct septin rings in a single cell by Elm1p and Gin4p kinases, Mol. Biol. Cell, 2009, 20, 2311-2326

[114] Finley K.R., Berman J., Microtubules in Candida albicans hyphae drive nuclear dynamics and connect cell cycle progression to morphogenesis, Eukaryot. Cell, 2005, 4, 1697-1711

[115] Westfall P.J., Momany M., Aspergillus nidulans septin AspB plays pre- and postmitotic roles in septum, branch, and conidiophore development, Mol. Biol. Cell, 2002, 13, 110-118

[116] Boyce K.J., Chang H., D'Souza C.A., Kronstad J.W., An Ustilago maydis septin is required for filamentous growth in culture and for full symptom development on maize, Eukaryot. Cell, 2005, 4, 2044-2056

[117] Canovas D., Perez-Martin J., Sphingolipid biosynthesis is required for polar growth in the dimorphic phytopathogen Ustilago maydis, Fungal Genet. Biol., 2009, 46, 190-200

[118] Berlin A., Paoletti A., Chang F., Mid2p stabilizes septin rings during cytokinesis in fission yeast, J. Cell Biol., 2003, 160, 1083-1092

[119] Tasto J.J., Morrell J.L., Gould K.L., An anillin homologue, Mid2p, acts during fission yeast cytokinesis to organize the septin ring and promote cell separation, J. Cell Biol., 2003, 160, 1093-1103

[120] Dekker N., Speijer D., Grun C.H., van den Berg M., de Haan A., Hochstenbach F., Role of the $\alpha$-glucanase Agn1p in fission-yeast cell separation, Mol. Biol. Cell, 2004, 15, 39033914

[121] Martín-Cuadrado A.B., Dueñas E., Sipiczki M., Vázquez de Aldana C.R., Rey F.del, The endo- $\beta-1,3$-glucanase eng $1 \mathrm{p}$ is required for dissolution of the primary septum during cell separation in Schizosaccharomyces pombe, J. Cell Sci., 2003, 116, 1689-1698

[122] Martín-Cuadrado A.B., Morrell J.L., Konomi M., An H., Petit C., Osumi M., et al., Role of septins and the exocyst complex in the function of hydrolytic enzymes responsible for fission yeast cell separation, Mol. Biol. Cell, 2005, 16, 4867-4881

[123] Petit C.S., Mehta S., Roberts R.H., Gould K.L., Ace2p contributes to fission yeast septin ring assembly by regulating mid $^{+}$expression, J. Cell Sci., 2005, 118, 5731-5742

[124] Dong Z., Ferger B., Paterna J.C., Vogel D., Furler S., Osinde M., et al., Dopaminedependent neurodegeneration in rats induced by viral vector-mediated overexpression of the parkin target protein, CDCrel-1, Proc. Natl. Acad. Sci. USA, 2003, 100, 12438-12443 
[125] Kinoshita A., Kinoshita M., Akiyama H., Tomimoto H., Akiguchi I., Kumar S., et al., Identification of septins in neurofibrillary tangles in Alzheimer's disease, Am. J. Pathol., $1998,153,1551-1560$

[126] Kuhlenbaumer G., Hannibal M.C., Nelis E., Schirmacher A., Verpoorten N., Meuleman J., et al., Mutations in SEPT9 cause hereditary neuralgic amyotrophy, Nat. Genet., 2005, 37, 1044-1046 


\section{LEGEND TO FIGURES}

Figure 1: Septin dynamics in S. cerevisiae. A) The dynamics of septin subunits inside the ring is cell-cycle dependent. The only moments of fluidity in the ring coincide with topological changes in the plasma membrane, i.e. bud emergence and cytokinesis. Proteins known to be essential for septin assembly and dynamics are shown. Bottom bar represents the periods of "frozen" (white) or "fluid" (grey) septin behaviour along the cell cycle. B) The septin ring itself is dynamic. Septin collar (hourglass) division into two rings (left panel) is accompanied by a $90^{\circ}$ rotation in the alignment of septin filaments, which rotate from an arrangement parallel to the mother-bud axis to an arrangement parallel to the septum (right panel).

Figure 2: Schematic representation of the morphology of hyphae from the wild-type strain and $\operatorname{sep} 7 \Delta, \operatorname{ccn} 1 \Delta, \sec 3 \Delta$ and $C D C 11$-S394A mutants. Wild-type hyphae show a predominantly apical growth pattern, secretion being directed to the tip of the hyphae. sep $7 \Delta$ mutants assemble normal filaments, but their septin rings disassemble after cytokinesis and the hyphal compartments separate. $\operatorname{ccn} 1 \Delta, \sec 3 \Delta$ and $C D C 11-\mathrm{S} 394 \mathrm{~A}$ mutants have normal hyphal development until the first septum is assembled, after which a swollen distal compartment is formed. Septins are shown in green and septa in red, while arrows mark the direction of polarized secretion.

Figure 3: The yeast septin ring serves as a scaffold for $\mathrm{Cdc14}$, which is required for the localization of the transcription factor Ace 2 to the daughter nucleus. As a consequence, the cell separation program is activated and the septum is degraded. When cells initiate hyphal growth, septin ring dynamics changes to a "hypha-specific" state, in which Cdc10 rapidly exchanges from a cytoplasmic pool (a process dependent on the septin Sep7 and its phosphorylation mediated by Hgc1). This alters the scaffolding properties of the septin ring, preventing Cdc14 localization at the bud neck, which in turn finally blocks cell separation. Dashed lines, indirect regulation. The images show Cdc12-GFP localization (green) in yeast and hyphae stained with Calcofluor white (blue). 
Figure 4: Septins are essential for C. albicans hyphal development. During yeast growth, Cdc11 is phosphorylated by Gin4 at Ser395 at the end of the previous cell cycle. Upon hyphal induction, this phosphorylated residue serves as a priming signal for the recruitment of the Ccn1-Cdc28 complex that phosphorylates Ser394, allowing correct hyphal growth after the first septin ring. 
A

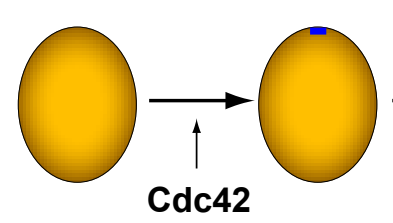

Cdc42

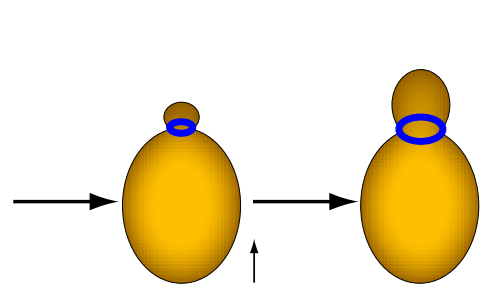

Cla4

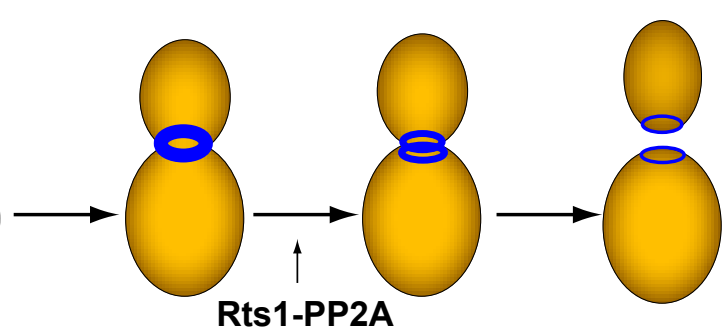

\begin{tabular}{|c|c|c|c|c|}
\hline Frozen & Fluid & Frozen & Fluid & Frozen \\
\hline
\end{tabular}

B

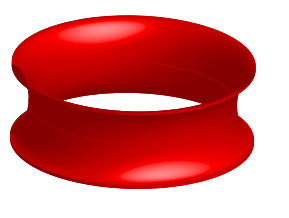

Collar

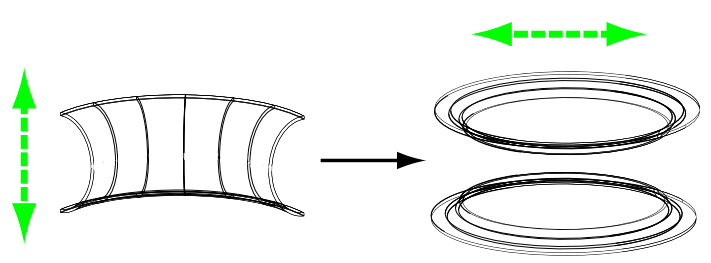

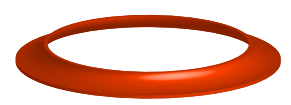

Divided rings 
Wild-type

rings

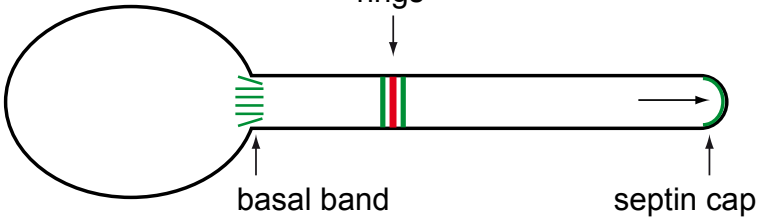

sep7 $\triangle$

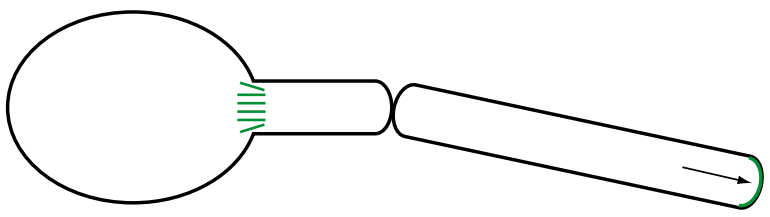

$\operatorname{ccn} 1 \Delta$, Cdc11-S394A, sec3 $\Delta$

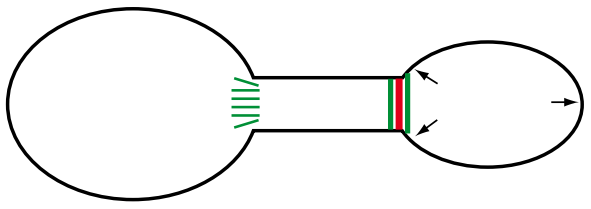



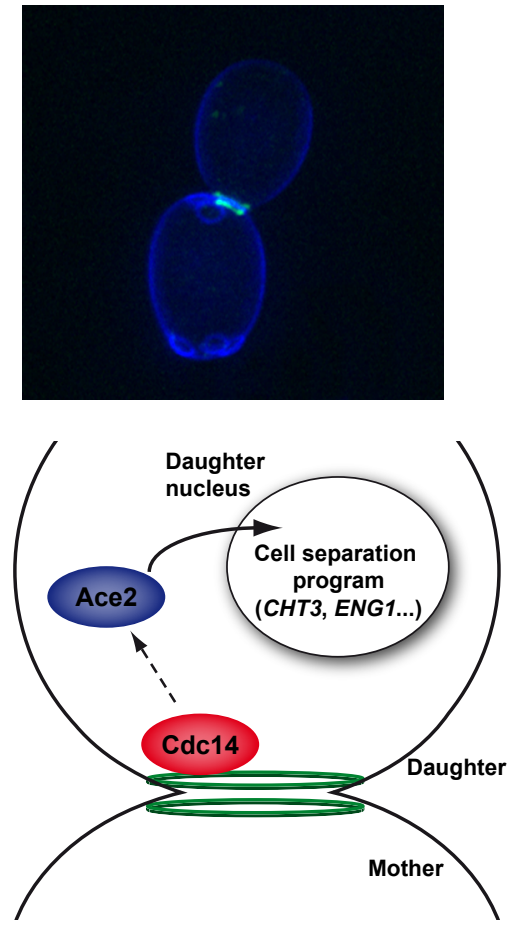

Hyphal induction

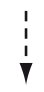
i Hgc1/Cdc28

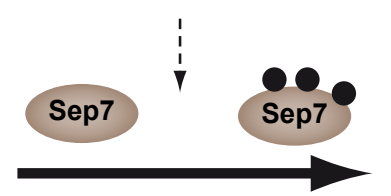

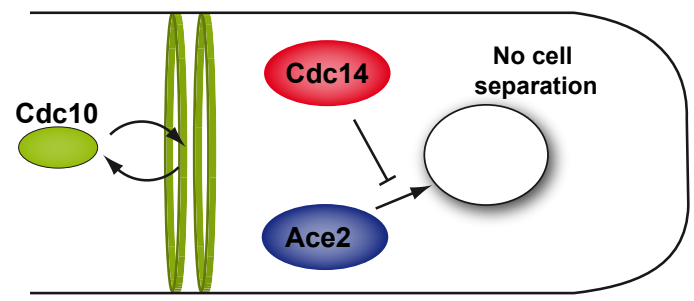




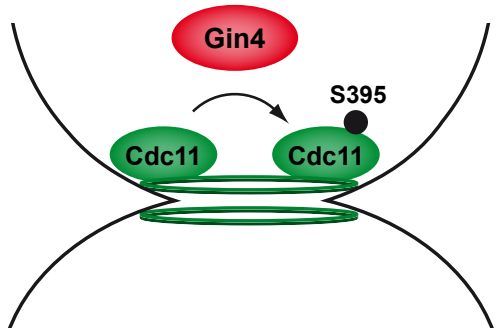

Hyphal induction

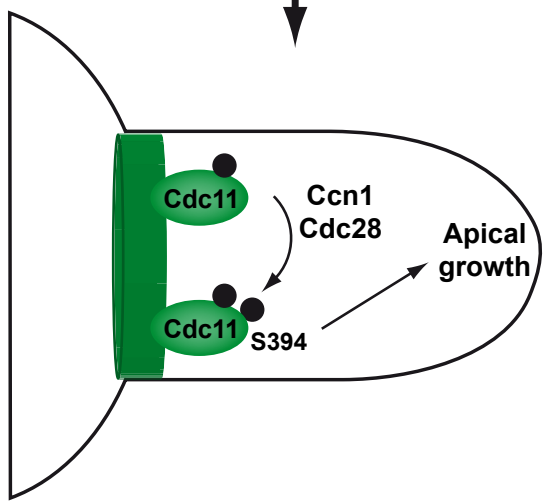

